\title{
Structural properties of dwarf ellipticals and the connection with (ordinary) elliptical galaxies
}

\author{
Alister W. Graham \\ Mount Stromlo and Siding Spring Observatories, Australian National University, Private Bag, \\ Weston Creek PO, ACT 2611, Australia. \\ email: Graham@mso.anu.edu.au
}

\begin{abstract}
This article reviews the popular reasons for the belief that dwarf elliptical galaxies and (ordinary) elliptical galaxies are distinct and separate species. They include: light-profile shape (or similarly image concentration); the magnitude-central surface brightness diagram; the magnitude-effective surface brightness diagram (or similarly the magnitude-effective radius diagram); and the Fundamental Plane. It is shown how a continuous trend between luminosity and a) light-profile shape, and b) central surface brightness (until the onest of core formation at $M_{B} \sim-20.5 \mathrm{mag}$ ), results in a unification of the dwarf elliptical and (ordinary) elliptical galaxies. Neither the above four reasons, nor the luminosity function (at least in the Virgo cluster) provide evidence for a division at $M_{B}=-18$ mag between the dwarf elliptical and (ordinary) elliptical galaxies. Instead, they appear to be continuous extensions of each other.
\end{abstract}

Keywords. galaxies: dwarf, galaxies: elliptical and lenticular, $\mathrm{cD}$, galaxies: formation, galaxies: fundamental parameters, galaxies: luminosity function, galaxies: structure

\section{Introduction}

For at least the past two decades, astronomers have believed that dwarf elliptical (dE) galaxies (not to be confused with dwarf spheroidal galaxies, which typically have absolute magnitudes fainter than $M_{B}=-13 \mathrm{mag}$ ) are distinct objects from the more luminous (ordinary) elliptical (E) galaxies. The divide between these allegedly different classes is supposed to occur at an absolute magnitude of $M_{B}=-18$ mag (e.g., Kormendy 1985).

In this talk I will review the most oftenly quoted reasons why the dEs are thought to be different from the Es, and then go on to explain why I think these past claims for a discontinuity at $M_{B}=-18$ mag should be reconsidered. I will argue that a continuity in structural properties exists across this alleged boundary.

Much, but not all, of this talk is based on Sections 1 and 4 from Graham \& Guzmán (2003), where further details may be found.

\section{Past evidence of continuity}

Before addressing the issue of galaxy structure, which is where past claims of discontinuity have their roots, it is relevant to review various other physical aspects of the $\mathrm{dE}$ and $\mathrm{E}$ population. Several physical quantities vary smoothly across the alleged division at $-18 B$-mag.

The average globular cluster metallicity is known to vary continuously, as a function of host galaxy magnitude, across the alleged dE/E divide (e.g., Forbes et al. 1996, their figure 14). Côté (2005, these proceedings) has shown how this arises from a systematic 
variation in the relative numbers of red and blue globular clusters, as opposed to a single population changing its mean metallicity.

Furthermore, the host galaxies themselves follow a luminosity-metallicity relation that shows no sign of a division or break at $M_{B}=-18$ mag (e.g., Caldwell \& Bothun 1987). Related to this, the colour-magnitude relation shows no sign of a division at $M_{B}=-18$ mag (e.g., Caldwell 1983). Now, on average, dEs do have a different metallicity from the brighter Es, but the key point is that there is a continuous change in chemical composition between the two. If one was to measure the average height and weight of a sample of adults, and then do the same for a sample of children, one would of course conclude that these populations have different physical properties. But this obviously tells us little as to whether or not children are the same specie as adults.

Aside from the stellar populations not knowing about the supposed $\mathrm{dE} / \mathrm{E}$ transition, there is also no dynamical evidence. Including dozens of dEs, Matković \& Guzmán (2005, and also these proceedings) and De Rijcke et al. (2005, see also these proceedings) have both shown that the luminosity-velocity dispersion $(L-\sigma)$ relation continues linearly across the alleged boundary between the dEs and Es. Interestingly, both studies found that the slope of this relation is $\sim 2$, jumping to $\sim 4$ and thus recovering the Faber-Jackson (1976) relation at magnitudes greater than $\sim-20.5 B$-mag.

What then, are the popular reasons which have led people to believe that the dEs form a uniquely different class of object to the Es?

\section{Past evidence of discontinuity}

This section lists the three most often quoted reasons why dwarf and giant elliptical galaxies are thought to be different species of object. The reasons pertain to galaxy structure. In the light of new data, however, the following section will explain why these three arguments are no longer valid. The following section will also counter two further claims of support for a $\mathrm{dE} / \mathrm{E}$ discontinuity at $M_{B}=-18 \mathrm{mag}$.

(i) Dwarf elliptical galaxies have exponential light-profiles (Faber \& Lin 1983; Binggeli, Sandage \& Tarenghi 1984), while elliptical galaxies have $R^{1 / 4}$ profiles (de Vaucouleurs 1948, 1959; de Vaucouleurs \& Capaccioli 1979).

(ii) Dwarf elliptical galaxies define a distribution that is almost at right angles to that traced by the brighter elliptical galaxies in the diagram of central surface brightness, $\mu_{0}$, versus absolute magnitude, $M$, (Kormendy 1985, his figure 3). Such behaviour has been taken as evidence that different formation processes must have shaped the dEs and the Es. The divide is said to occur at an absolute magnitude of $-18 B$-mag.

(iii) In diagrams which plot a galaxy's effective surface brightness, $\mu_{\mathrm{e}}$, dwarf elliptical galaxies follow a distribution that is clearly offset to that defined by the brighter elliptical galaxies. This is the case for the $M-\mu_{\mathrm{e}}$ and $R_{\mathrm{e}}-\mu_{\mathrm{e}}$ diagram (Capaccioli, Caon \& D'Onofrio 1992, their figure 4; Binggeli \& Ferguson 1994; their section 2.2.2), and also for the Fundamental Plane (Djorgovski \& Davis 1987) when constructed using effective surface brightnesses (e.g., Geha, Guhathakurta \& van der Marel 2002).

\section{A unified picture}

In the Author's opinion, there have been two key advances since the above three observations were established. The first has come from a closer scrutiny of optical lightprofiles. Studies such as those by Schombert (1986) revealed that the $R^{1 / 4}$-law was not as universal as previously thought. Similarly, analyses by Caldwell \& Bothun (1987) and Binggeli \& Cameron (1991) revealed that the dEs have magnitude-dependent departures 


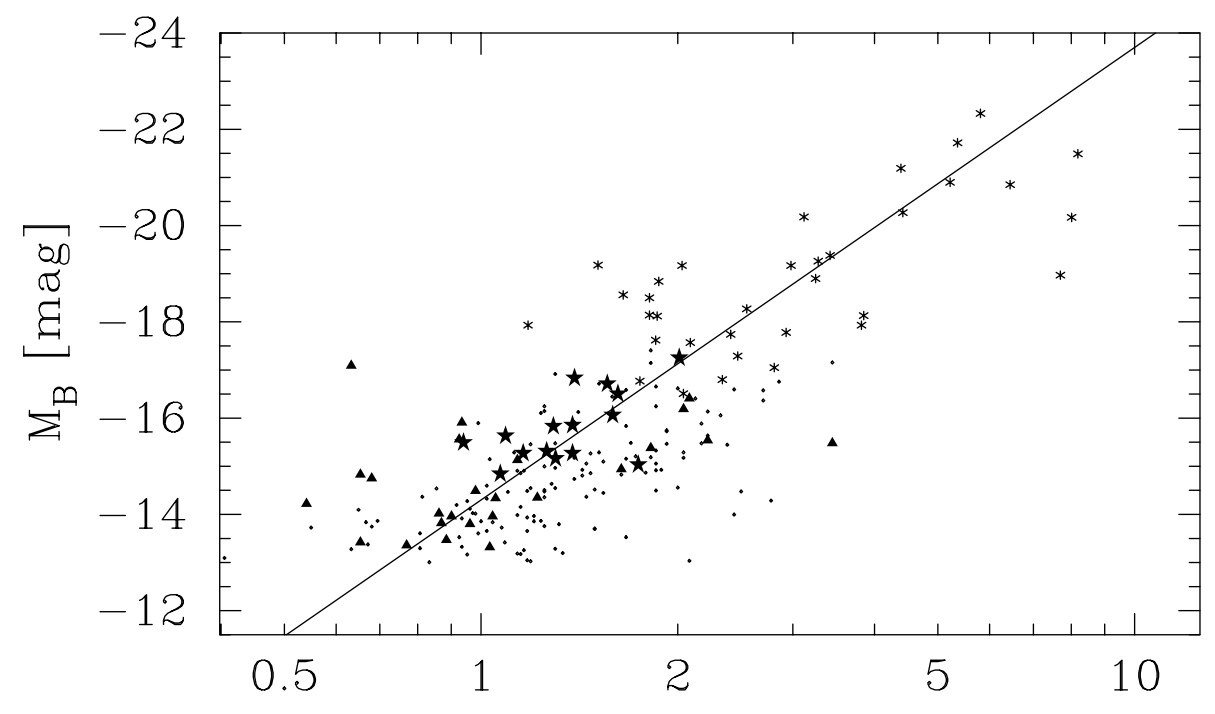

$\mathrm{n}$

Figure 1. Absolute $B$-band galaxy magnitude versus the logarithm of the Sérsic shape index $n$. Due to biasing from the magnitude cutoff at $M_{B} \sim-13 \mathrm{mag}$, the line $M_{B}=-9.4 \log (n)-14.3$ has been estimated by eye. Dots represent dE galaxies from Binggeli \& Jerjen (1998), triangles are dE galaxies from Stiavelli et al. (2001), large stars are dE galaxies from Graham \& Guzmán (2003), asterix are intermediate to bright E galaxies from Caon et al. (1993) and D'Onofrio et al. (1994). Lenticular galaxies have been excluded.

from the exponential model. The subsequent use of high-quality CCD imagery no doubt helped to refine this picture. Second, the superb resolution provided by the Hubble Space Telescope has allowed us to probe the centers of galaxies on scales previously washed out by atmospheric seeing. The impact of these two things on the above three points is given below.

(i) There is no question that de Vaucouleurs' $R^{1 / 4}$ model fits many elliptical galaxies remarkably well. It is a clever and useful empirical function. However, as noted in Djorgovski \& Kormendy (1989), it fits best elliptical galaxies with $M_{B} \sim-21 \mathrm{mag}$; brighter and fainter galaxies having different curvature than described by the $R^{1 / 4}$ model. Thanks to the pioneering work of Capaccioli and collaborators, most notably Caon, Capaccioli \& D'Onofrio (1993), numerous studies have now shown that Sérsic's (1963, 1968) $R^{1 / n}$ generalisation $\dagger$ accommodates for the real (Trujillo, Graham \& Caon 2001; their section 2) and systematic changes in light-profile shape $n$ with absolute magnitude (e.g., D’Onofrio, Capaccioli, \& Caon 1994; Graham et al. 1996; Vennik et al. 1996). Similarly, the stellar distribution in dwarf elliptical galaxies is also a function of absolute magnitude (e.g., Davies et al. 1988; Cellone, Forte, \& Geisler 1994; Vennik \& Richter 1994; Young \& Currie 1994, 1995; Karachentseva et al. 1996; Jerjen \& Binggeli 1997). This is shown in Figure 1 using a heterogeneous sample of literature data. Presumably the actual level of scatter is less than seen here. One may alternatively plot concentration, rather than profile shape, as done in Graham, Trujillo \& Caon (2001).

(ii) Using HST data from the Nuker team (Faber et al. 1997), the $M-\mu_{0}$ relation is now much better defined than it was twenty years ago, especially at the bright end. Furthermore, the increased number of galaxies around the alleged transition magnitude of -18 $B$-mag has also improved our understanding of what is going on. What is immediately

$\dagger$ A useful compilation of various Sérsic expressions can be found in Graham \& Driver (2005). 


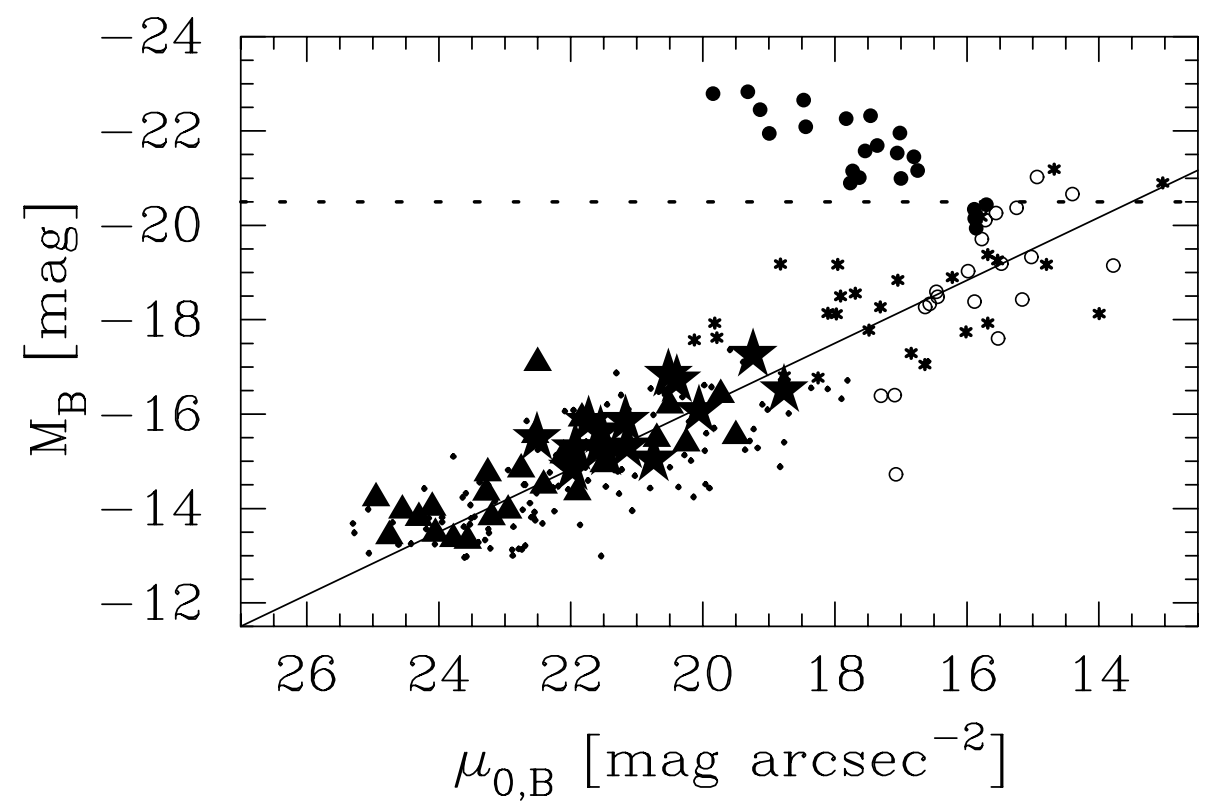

Figure 2. Galaxy magnitude versus (host galaxy) central surface brightness. Due to biasing from the magnitude cutoff at $M_{B} \sim-13 \mathrm{mag}$, the line $M_{B}=(2 / 3) \mu_{0}-29.5$ has been estimated by eye rather than from a linear regression routine. The central surface brightness values obtained from Sérsic models fitted to luminous $\left(M_{B} \lesssim-20.5 \mathrm{mag}\right) \mathrm{E}$ galaxies follow this same relation (Jerjen \& Binggeli 1997), although they were not used to define it. The symbols are the same as in Figure 1, with the additional open circles representing the so-called "power-law" E galaxies from Faber et al. (1997), and the filled circles representing the "core" E galaxies from these same authors.

clear from Figure 2 is that there is no break at $M_{B}=-18$ mag. Instead, a linear trend exists across this supposed divide, and extends from $M_{B}=-13$ mag to $M_{B} \sim-20.5$ mag. This diagram does not imply that the dEs $\left(M_{B}>-18 \mathrm{mag}\right)$ experienced a different formation process to the Es.

The behaviour of the elliptical galaxies in Figure 2 is similar to that seen in the $L-\sigma$ diagram, in which galaxies more luminous than $M_{B} \sim-20.5$ mag depart from the linear distribution defined by the less luminous Es and dEs (see Matković \& Guzmán 2005, these proceedings, their figure 1).

The deviant galaxies at the high-luminosity end in Figure 2 have all been identified by the Nuker team as "core" galaxies: their central light-profiles display a clear break and downward flattening relative to the inward extrapolation of their outer (Sérsic) stellar distribution (Graham et al. 2003; Trujillo et al. 2004). Such "core" galaxies may have formed from the dissipationless merger of two or more lesser Es, in which the progenitor galaxy's supermassive black holes have coalesced at the center of the new galaxy via the gravitational slingshot, and thus evacuation, of the central stars (Begelman, Blandford, \& Rees 1980; Ebisuzaki, Makino, \& Okumura 1991). This local, rather than global, physical process, reduces the central flux by $\sim 0.1 \%$ of the total galaxy flux (Graham 2004).

It is worth noting that the central surface brightness one would obtain from the inward extrapolation of the undisturbed outer light-profile of the "core" galaxies yields values that fall on the linear relation shown in Figure 2 (Jerjen \& Binggeli 1997).

(iii) The difference between the central surface brightness, $\mu_{0}$, and the effective surface brightness, $\mu_{\mathrm{e}}$ (the surface brightness at the effective half-light radius $R_{\mathrm{e}}$ ), is the same 


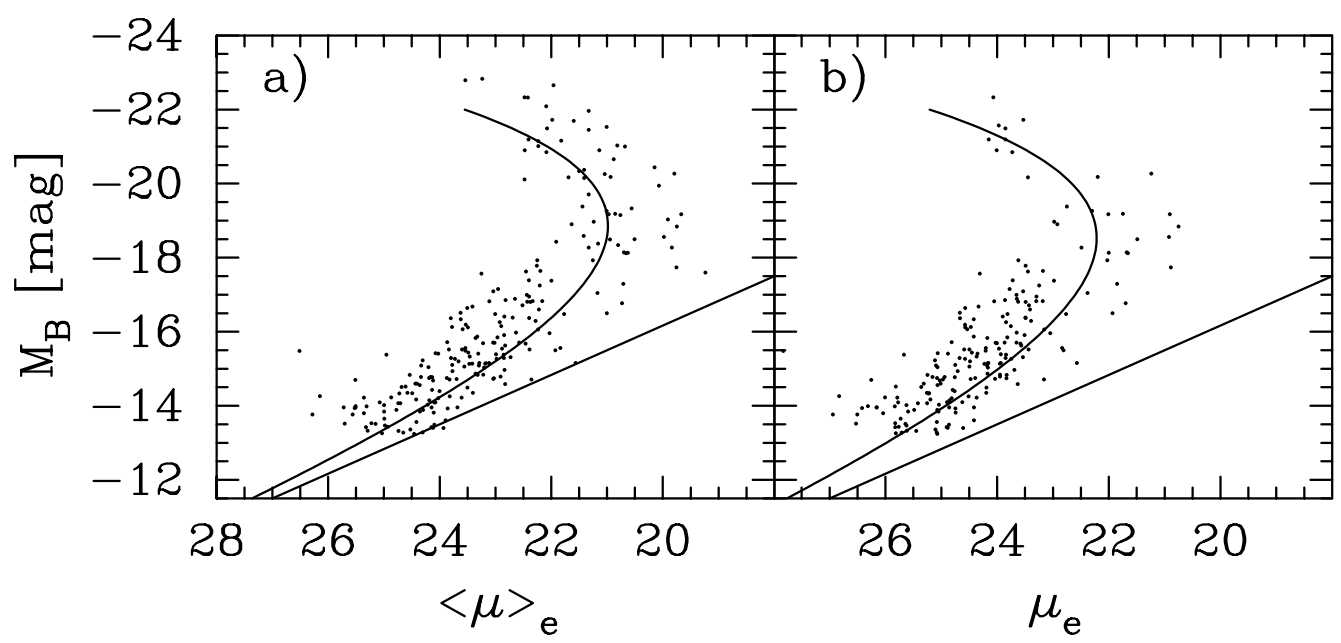

Figure 3. The curved lines show the expected behavior of galaxies in the (a) magnitude-mean effective surface brightness and (b) magnitude-effective surface brightness diagrams given the linear relations seen in Figures 1 and 2. The straight line shown here is from Figure 2.

for every $R^{1 / 4}$ profile. This difference, however, varies as a function of profile shape $n$, and is given by the expression $\mu_{\mathrm{e}}=\mu_{0}+1.086 b$, where $b \sim(2 n-1 / 3)$. So, as the galaxy magnitudes brighten in Figure 2, the value of $n$ increases (Figure 1) and thus the value of $\mu_{\mathrm{e}}-\mu_{0}$ increases. This is shown in Figure $3 \mathrm{~b}$. Related to this, the mean surface brightness within the effective radius, $\langle\mu\rangle_{\mathrm{e}}$, can be derived from the expression $\langle\mu\rangle_{\mathrm{e}}=\mu_{\mathrm{e}}-2.5 \log \left[e^{b} n \Gamma(2 n) / b^{2 n}\right]$, where $\Gamma$ is the gamma function (e.g., Graham \& Driver 2005).

The different slopes for the $\mathrm{dE}$ and $\mathrm{E}$ galaxy distributions in Figure 3 is merely a consequence of a continuously varying profile shape with galaxy luminosity, it does not imply distinctly different galaxy formation processes for dEs and Es.

\subsection{Other issues}

(a) Kormendy (2004) has claimed that the decline in the dE galaxy luminosity function at bright magnitudes, and the associated decline in the E galaxy luminosity function at faint magnitudes is evidence that they are distinct populations. This argument of course falls down if one assigns a galaxy to the $\mathrm{dE}$ or $\mathrm{E}$ population based on its luminosity. Similarly, if one assigns an object to a class based on its appearance (i.e., using image concentration or light-profile shape), the situation remains unchanged (see Figure 1).

Figure 4 shows the luminosity function of the Virgo dE + E galaxies. There is obviously nothing special about $M_{B}=-18 \mathrm{mag}$, and certainly no evidence for a deficit of galaxies at this magnitude, nor is it the overlap region of two distributions peaking at fainter and brighter magnitudes.

(b) From the beginning it has been known that galaxies with central velocity dispersions less than about $100 \mathrm{~km} \mathrm{~s}^{-1}$ do not follow the "Fundamental Plane" (Djorgovski \& Davis 1987) as defined by luminous elliptical galaxies.

Using $L=2 \pi R_{\mathrm{e}}^{2}\langle I\rangle_{\mathrm{e}}$, it can be shown that the same mechanism that causes the curved relations in Figure 3 is also behind the different slopes in the $\langle\mu\rangle_{\mathrm{e}}-\log R_{\mathrm{e}}$ and $M-\log R_{\mathrm{e}}$ diagram for the dEs and Es. It therefore comes as no surprise that the dEs are not distributed on the Fundamental Plane. As was noted in Graham \& Guzmán (2004), the Fundamental Plane is simply the tangent sheet to the high-luminosity end of a curved surface in the three-space of $\langle\mu\rangle_{\mathrm{e}}, R_{\mathrm{e}}$, and $\sigma$. Only bright Es that follow the 


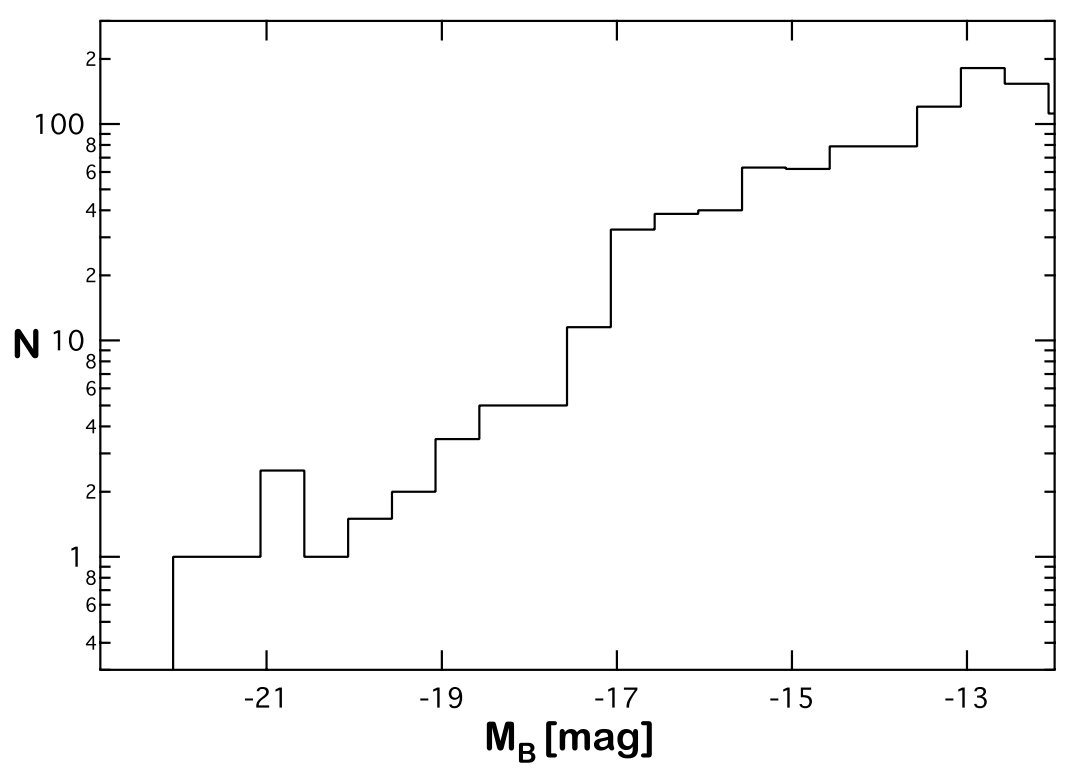

Figure 4. Virgo cluster $\mathrm{dE}+\mathrm{E}$ galaxy luminosity function. There is no evidence for a division at $M_{B}=-18 \mathrm{mag}$. Likely ( $50 \%$ chance) cluster members are weighted by 0.5 . Figure courtesy of H. Jerjen.

Kormendy (1977) relation (see Capaccioli et al. 1992, their figure 4) will reside on typical constructions of the Fundamental Plane. The use of central surface brightness mitigates the apparent different behaviour of the dEs and Es (Guzmán et al. 2005, in prep).

\section{Conclusions}

Chemically and dynamically, the dEs appear to form a continuous extension with the Es. Both the colour-magnitude relation (but see Lisker et al., these proceedings) and the $L-\sigma$ relation show a continuous linear trend across the alleged boundary at $-18 B-$ mag. Similarly, the $\mathrm{dE}+\mathrm{E}$ galaxy luminosity function shows no evidence of a divide at this magnitude.

In terms of galaxy structure, $\mathrm{dEs}$ and Es display a continuous range of properties that vary smoothly across the alleged $\mathrm{dE} / \mathrm{E}$ divide. The shape of their (projected) stellar distribution, quantified through Sérsic's index $n$, increases steadily with luminosity from values around 0.5 for the fainter dwarfs, to values greater than 4 for the more luminous ellipticals. The central (host galaxy) surface brightness brightens linearly (from $M_{B} \sim$ $-13 \mathrm{mag}$ ) as the host galaxy magnitude brightens. There is no discontinuity at $M_{B}=-18$ mag in the $M-\mu_{0}$ diagram.

The above two relations account for the near-orthogonal distribution of dEs and Es in diagrams of effective surface brightness versus (i) magnitude and (ii) effective radius. They also explain why the dEs depart from the Es in the traditional construction of the Fundamental Plane, which uses effective surface brightness.

We report a clear break in the $M-\mu_{0}$ relation at $M_{B} \sim-20.5 \mathrm{mag}$, such that more luminous galaxies have fainter central surface brightnesses. Thanks to the resolution of $H S T$, this departure of the brightest elliptical galaxies from the main $M-\mu_{0}$ relation is understood to be associated with the presence of partially depleted cores. 


\section{Acknowledgements}

I wish to thank my collaborators Rafael Guzmán, Ana Matković and Ileana Vass who have contributed to various aspects of this paper. I am also grateful to Helmut Jerjen who provided the luminosity function shown in Figure 4. This research was supported in part by NASA grant HST-AR-09927.01-A from the Space Telescope Science Institute, which is operated by the Association of Universities for Research in Astronomy, Inc., under NASA contract NAS5-26555.

\section{References}

Begelman, M.C., Blandford, R.D. \& Rees, M.J. 1980, Nature 287, 307

Binggeli, B. \& Cameron, L.M. 1991, A\&A 252, 27

Binggeli, B. \& Jerjen, H. 1998, A\&\&A 333, 17

Binggeli, B., Sandage, A. \& Tarenghi, M. 1984, AJ 89, 64

Caldwell, N. 1983, $A J$ 88, 804

Caldwell, N. \& Bothun, G.D. 1987, AJ 94, 1126

Caon, N., Capaccioli, M. \& D'Onofrio, M. 1993, MNRAS 265, 1013

Capaccioli, M., Caon, N. \& D'Onofrio, M. 1992, MNRAS 259, 323

Cellone, S.A., Forte, J.C., \& Geisler, D. 1994, ApJS 93, 397

Davies, J.I., Phillipps, S., Cawson, M.G.M., Disney, M.J. \& Kibblewhite, E.J. 1988, MNRAS 232,239

De Rijcke, S., Michielsen, D., Dejonghe, H., Zeilinger, W.W. \& Hau, G.K.T. 2005, $A \mathscr{E} A$ in press (astro-ph/0412553)

de Vaucouleurs, G. 1948, Annales d'Astrophysique 11, 247

de Vaucouleurs, G. 1959, in: S. Flügge (ed.) Handbuch der Physik (Berlin: Springer) p. 275

de Vaucouleurs, G. \& Capaccioli, M. 1979, ApJS 40, 669

Djorgovski, S. \& Davis, M. 1987, ApJ 313, 59

Djorgovski, S. \& Kormendy, J. 1989, ARA\&A 27, 235

D’Onofrio, M., Capaccioli, M. \& Caon, N. 1994, MNRAS 271, 523

Ebisuzaki, T., Makino, J. \& Okumura, S.K. 1991, Nature 354, 212

Faber, S.M. \& Jackson, R.E. 1976, ApJ 204, 668

Faber, S.M. \& Lin, D.M.C. 1983, ApJ 266, L17

Faber, S.M., et al. 1997, AJ 114, 1771

Ferguson, H.C. \& Binggeli, B. 1994, A\&ARv 6, 67

Forbes, D.A., Franx, M., Illingworth, G.D. \& Carollo, C.M. 1996, ApJ 467, 126

Geha, M., Guhathakurta, P. \& van der Marel, R.P. 2002, AJ, 124, 3073

Graham, A.W. 2004, ApJ 613, L33

Graham, A.W. \& Driver, S.P. 2005, PASA 22(2), 118

Graham, A.W, Erwin, P., Trujillo, I. \& Asensio-Ramos, A. 2003, AJ 125, 2951

Graham, A.W. \& Guzmán, R. 2003, AJ 125, 2936

Graham, A.W. \& Guzmán, R. 2004, in: D.L. Block, I. Puerari, K.C. Freeman, R. Groess \& E.K. Block (eds.), Penetrating bars through masks of cosmic dust, (Dordrecht: Springer), vol. 319 , p. 723

Graham, A.W., Lauer, T., Colless, M.M. \& Postman, M. 1996, ApJ 465, 534

Jerjen, H., \& Binggeli, B. 1997, in: The Nature of Elliptical Galaxies; The Second Stromlo Symposium, ASP Conf. Ser., vol. 116, p. 239

Karachentseva, V.E., Prugniel, P., Vennik, J., Richter, G.M., Thuan, T.X. \& Martin, J.M. 1996, A\&ASS 117, 343

Kormendy, J. 2004, in: D.L. Block, I. Puerari, K.C. Freeman, R. Groess \& E.K. Block (eds.), Penetrating bars through masks of cosmic dust, (Dordrecht: Springer), vol. 319, p. 728

Kormendy, J. 1977, ApJ 218, 333

Kormendy, J. 1985, ApJ 295, 73

Matković, A. \& Guzmán, R. 2005, MNRAS, submitted

Schombert, J.M. 1986, ApJS 60, 603 
Sérsic, J.-L. 1963, Boletin de la Asociacion Argentina de Astronomia vol. 6, p. 41

Sérsic, J.-L. 1968, Atlas de Galaxias Australes (Cordoba: Observatorio Astronomico)

Stiavelli, M., Miller, B.W., Ferguson, H.C., Mack, J., Whitmore, B.C. \& Lotz, J.M. 2001, AJ 121,1385

Trujillo, I., Erwin, P., Asensio Ramos, A. \& Graham, A.W. 2004, AJ 127, 1917

Trujillo, I., Graham, A.W. \& Caon, N. 2001, MNRAS 326, 869

Vennik, J., Hopp, U., Kovachev, B., Kuhn, B. \& Elsässer, H. 1996, A\&ASS 117, 261

Vennik, J. \& Richter, G.M. 1994, Astron. Nachr. 315, H3, 245

Young, C.K. \& Currie, M.J. 1994, MNRAS 268, L11

Young, C.K. \& Currie, M.J. 1995, MNRAS 273, 1141

\section{Discussion}

TAYLOR: What are the smallest black holes you need in this picture to scour out the cored galaxies?

GrahAm: Bearing in mind that it's not simply the black hole masses, but whether the galaxy merger is dissipationless or not - as gas can facilitate black hole coalescence and also help repopulate the loss cone - the smallest black holes in galaxies with cores have masses around $10^{8} M_{\odot}$.

Conselice: The low-luminosity ellipticals also have black holes, and probably also form through mergers. If this is the case, as seems likely, then why don't the low-luminosity ellipticals also have depleted cores? Does this suggest that giant and fainter ellipticals really do have two formation methods?

Graham: A good question. Giant elliptical galaxies with partially depleted cores tend to be brighter than $\sim-20.5 B$-mag, while "power-law" galaxies tend to be fainter. It has been suggested that the "core" galaxies formed via a dissipationless merger event while the "power-law" galaxies formed from a relatively gas rich merger. No division at $-18 B$-mag is apparent between the "power-law" ellipticals and the dwarf ellipticals. In passing I note that the boxy/disky isophote divide also occurs at $\sim-20.5 B$-mag, as does the change in slope of the $L-\sigma$ relation (Matković \& Guzmán and de Rijcke et al. these proceedings). 